\title{
Monte Carlo simulations of Wyoming sodium montmorillonite hydrates
}

\author{
M. Chávez-Páez and K. Van Workum \\ Department of Chemical Engineering, University of Wisconsin-Madison, 1415 Engineering Drive, \\ Madison, Wisconsin 53706 \\ L. de Pablo \\ Instituto de Geología, Universidad Nacional Autónoma de México, México \\ J. J. de Pablo \\ Department of Chemical Engineering, University of Wisconsin-Madison, 1415 Engineering Drive, \\ Madison, Wisconsin 53706
}

(Received 6 June 2000; accepted 14 September 2000)

\begin{abstract}
Monte Carlo simulations have been used to predict the interlayer basal separations of sodium-saturated Wyoming clays at constant stress $\left(N P_{z z} T\right.$ ensemble) and at constant chemical potential ( $\mu V T$ ensemble). These simulations use the Ewald summation technique to incorporate long-range Coulombic interactions in the calculation of the total potential energy and the pressure tensor. A comparison is made between the use of one, two, and three sheets of clay. It is shown that, for small separations, at least two separate clay sheets must be used to avoid system-size effects. The stable interlamellar separations are determined by combining results from isostress-isothermal and grand canonical simulations. It is shown that, consistent with experiments, at the temperature and pressure studied here, the cations in the interlayer are hydrated, except at the smallest basal separations. (C) 2001 American Institute of Physics. [DOI: 10.1063/1.1322639]
\end{abstract}

\section{INTRODUCTION}

Clay minerals are the major components of soils. ${ }^{1}$ They consist of alumina silicate crystallites of colloidal dimensions usually stacked in layers. ${ }^{2}$ A single particle typically has lateral dimensions of $10^{3}$ to $10^{6} \AA$ and a thickness of about $10 \AA$. Their colloidal character and their large surface area-to-mass ratio give clay minerals a unique set of properties, which include highly reactive surfaces, high cation exchange capacities, catalytic activity, and the ability to swell when exposed to water. ${ }^{2}$ Clay minerals are industrially important in cosmetics, oil drilling, toxic and radioactive waste disposal, catalysis, drug and agrochemical delivery, and as additives for polymeric materials.

Clay minerals are phyllosilicates. The building blocks are two-dimensional arrays of silicon-oxygen tetrahedra and two-dimensional arrays of aluminum or magnesium oxygenhydroxyl octahedra. These sheets can be superimposed in different manners. Clay minerals formed by one tetrahedral sheet and one octahedral sheet are termed 1:1 clay minerals. If two tetrahedral sheets and one octahedral sheet are present, the clay minerals are termed 2:1. The 2:1 clay minerals are called dioctahedral if two of the octahedral positions are filled by trivalent $\mathrm{Al}$ atoms, and trioctahedral if the three octahedral positions are filled by three divalent $\mathrm{Mg}$ atoms. The electroneutral 2:1 dioctahedral clay mineral is termed pyrophyllite, whereas the mineral talc is an electroneutral $2: 1$ trioctahedral mineral.

The structure of the minerals of the montmorillonite group, also called montmorillonoids or smectites, ${ }^{2}$ is derived from that of the pyrophyllite and talc by replacing tetravalent $\mathrm{Si}$ atoms by trivalent $\mathrm{Al}$ atoms in the tetrahedral sheet, and replacing trivalent $\mathrm{Al}$ atoms by divalent $\mathrm{Mg}$ atoms in the octahedral sheet. These substitutions yield an excess of negative charge in the clay sheet, which is compensated by the adsorption of cations such as sodium onto the surface of the clay. Montmorillonites can adsorb water between the layers; many of the properties of montmorillonites are related to their ability to exchange water and cations with the surrounding media. This behavior should be contrasted with that of neutral minerals, such as pyrophyllite and talc, which do not swell when exposed to water. It is therefore of interest to examine the swelling of clays, how it arises, and to determine the state of hydration of cations as a function of water content.

In this work we investigate the equilibrium properties of smectites or expanding clays. These clays have the capacity to capture water molecules and hydrated ions in the interlayer space, thereby generating powerful stresses that force the clay layers apart and increase the overall volume of the mineral. In particular, in this study we are interested in the swelling of hydrated clay minerals both as a function of the water content under constant external pressure and at constant chemical potential. We take as our clay model system the sodium-saturated Wyoming-type montmorillonite. ${ }^{3}$ Wyoming montmorillonite is characterized by a layer charge of $Q=0.85$, or less, per $\mathrm{O}_{20}(\mathrm{OH})_{4}$ formula unit cell. Tetrahedral substitutions are responsible for $15 \%$ to $50 \%$ of the total charge. In particular, we will consider the unit cell formula $\mathrm{Na}_{0.75} n \mathrm{H}_{2} \mathrm{O}\left(\mathrm{Si}_{7.75} \mathrm{Al}_{0.25}\right)\left(\mathrm{Al}_{3.5} \mathrm{Mg}_{0.5}\right) \mathrm{O}_{20}(\mathrm{OH})_{4}$, which represents a mineral with charge $Q=0.75$, with $33 \%$ of the charge in the tetrahedral sheets. As explained later, this formula indicates that our clay layer contains eight unit cells with four isomorphous substitutions of trivalent $\mathrm{Al}$ atoms by divalent $\mathrm{Mg}$ atoms in the octahedral sheet, two isomorphous substi- 
tutions of tetravalent $\mathrm{Si}$ atoms by trivalent $\mathrm{Al}$ atoms in the tetrahedral sheet, and six compensating monovalent sodium ions in the interlayer region.

Several Monte Carlo and molecular dynamics simulation studies of the swelling behavior of clays have appeared in the literature. ${ }^{4-8}$ The results of these studies have sometimes been contradictory. The purpose of this work is to present a more extensive simulation study of the swelling of sodium montmorillonite and to attempt to clarify some of the discrepancies in previous work. For this, we use simulations in the $N P_{z z} T$ and $\mu V T$ ensembles. ${ }^{9}$ In $N P_{z z} T$ simulations, the number of molecules in the system, the stress, and the temperature are constant, whereas in $\mu V T$ simulations the chemical potential, the volume, and the temperature are constant. The structure and partial charges of the model clay are taken from Skipper et al. ${ }^{5}$ The interaction parameters for the clay-water system are based on the TIP4P and MCY models of water, ${ }^{10,11}$ and are given in Refs. 5 and 12. The remainder of this manuscript is organized as follows: Sec. II presents the methodology employed in our simulations. The interaction potentials and pressure tensor are presented in that section. The ensembles employed in this work are also discussed in Sec. II, along with simulation details. In Sec. III we discuss our results and compare them to previous work. In Sec. IV we summarize the main results of this work.

\section{METHODOLOGY}

\section{A. Potentials}

Previous studies of clay-water interactions in hydrated clay minerals ${ }^{4,5}$ have been based on the MCY and the TIP4P models of water. ${ }^{10,11}$ Based on these models, Skipper et al. expressed the potential energy of site-site interactions through the generic formula

$$
U_{i j}=U_{i j}^{\mathrm{Coul}}+U_{i j}^{\mathrm{LJ}},
$$

where electrostatic interactions are given by

$$
U_{i j}^{\text {Coul }}=\sum_{a=1}^{m_{i}} \sum_{b=1}^{m_{j}} \frac{q_{i a} q_{j b}}{r_{i a j b}}
$$

and (LJ) Lennard-Jones-type or dispersion contributions are given by

$$
\begin{aligned}
& U_{i j}^{\mathrm{LJ}}=\sum_{a=1}^{m_{i}} \sum_{b=1}^{m_{j}}\left[-A_{i a j b} e^{-B_{i a j b} r_{i a j b}}+C_{i a j b} e^{-D_{i a j b} r_{i a j b}}\right. \\
& \left.-\frac{E_{i a j b}}{\left(r_{i a j b}\right)^{6}}+\frac{F_{i a j b}}{\left(r_{i a j b}\right)^{12}}\right] \text {. }
\end{aligned}
$$

In the above equations $q_{i a}$ is the charge on site $a$ of molecule $i, r_{i a j b}$ is the distance between sites $a$ and $b$ located on molecules $i$ and $j$, respectively, and $m_{i}$ is the number of sites in molecule $i$. The quantities $A, B, C, D, E$, and $F$ are site specific parameters which depend on the particular molecular model of water, i.e., MCY or TIP4P. The values of these parameters are given in the Appendix. Note that Eqs. (1), (2), and (3) are used for clay-water, water-water interactions, water-sodium, and clay-sodium interactions. Under periodic boundary conditions, the electrostatic energy can be cal- culated using Ewald sums ${ }^{9,13}$ (although other methods such as a reaction field could be used, ${ }^{9}$ provided the dielectric constant is sufficiently high), which efficiently take into account the long-range character of the Coulombic interactions. Under the Ewald summation formalism, the contribution to the total Coulombic energy $U_{\text {Coul }}$ can be written as $U_{\text {Coul }}=U_{\text {Dir }}+U_{\text {Rec }}+U_{\text {Self }}$, where

$$
\begin{aligned}
U_{\mathrm{Dir}} & =\frac{1}{2} \sum_{i \neq j} \sum_{a, b} \frac{q_{i a} q_{j b}}{r_{i a j b}} \operatorname{erfc}\left(\alpha r_{i a j b}\right), \\
U_{\mathrm{Rec}} & =\sum_{\mathbf{k} \neq 0} A(k) S(\mathbf{k}) S(-\mathbf{k})+\frac{2 \pi}{2 \epsilon+1}\left(\frac{\mathbf{M}^{2}}{V}\right),
\end{aligned}
$$

and

$$
U_{\text {Self }}=-\sum_{i, a}\left(\frac{\alpha}{\sqrt{\pi}}\left(q_{i a}\right)^{2}+\sum_{b>a} \frac{q_{i a} q_{i b} \operatorname{erf}\left(\alpha r_{i a i b}\right)}{r_{i a i b}}\right),
$$

where

$$
A(k)=\left(\frac{2 \pi}{V}\right) \frac{\exp \left[-k^{2} /\left(4 \alpha^{2}\right)\right]}{k^{2}},
$$

and

$$
S(\mathbf{k})=\sum_{i a} q_{i a} \exp \left(i \mathbf{k} \cdot \mathbf{r}_{i a}\right)
$$

The vector $\mathbf{M}$ is the total dipolar moment of the system, and $\epsilon$ is the dielectric constant of the surrounding medium. If the system is assumed to be immersed in a conductor, then $\epsilon$ $=\infty$ and the contribution from $\mathbf{M}$ vanishes (tin foil or conducting boundary condition), which is the convention we use in this work.

It has been shown ${ }^{14,15}$ that, for rigid molecules, the contribution of the real part of the Ewald sums to the pressure tensor, $\mathbf{P}$, can be evaluated from the virial and can be written as

$$
V\left(\mathbf{P}_{\text {Dir }}\right)_{\alpha \beta}=\sum_{i, a} \sum_{j>i, b}\left(\mathbf{r}_{i j}\right)_{\alpha}\left(\mathbf{f}_{i a j b}\right)_{\beta},
$$

where

$\mathbf{f}_{i a j b}=q_{i a} q_{j b}\left(\frac{\mathbf{r}_{i a j b}}{r_{i a j b}^{2}}\right)\left(\frac{\operatorname{erfc}\left(\alpha r_{i a j b}\right)}{r_{i a j b}}+\frac{2 \alpha}{\sqrt{\pi}} \exp \left[-\left(\alpha r_{i a j b}\right)^{2}\right]\right)$.

On the other hand, the contribution of the reciprocal part is given by

$$
\begin{aligned}
V\left(\mathbf{P}_{\mathrm{Rec}}\right)_{\alpha \beta}= & \sum_{\mathbf{k} \neq 0} A(k) S(\mathbf{k}) S(-\mathbf{k})\left(\delta_{\alpha \beta}-\frac{2 k_{\alpha} k_{\beta}}{k^{2}}-\frac{k_{\alpha} k_{\beta}}{2 \alpha^{2}}\right) \\
& +\sum_{\mathbf{k} \neq 0} 2 A(k)(\mathbf{k})_{\alpha} \sum_{i, a} q_{i a}\left(\mathbf{r}_{i a}-\mathbf{r}_{i}\right)_{\beta} \\
& \times \operatorname{Im}\left[S(\mathbf{k}) \exp \left(-i \mathbf{k} \cdot \mathbf{r}_{i a}\right)\right],
\end{aligned}
$$

where $\mathbf{r}_{i}$ is the position of the center-of-mass of molecule $i$. The second term in Eq. (11) represents the contribution to the pressure tensor that arises from the rigidity of the molecules. Note that these expressions are only for electrostatic 
TABLE I. Positions and charges assigned to the elements in the unit cell of the mineral pyrophyllite (dioctahedral 2:1 neutral clay), as reported by Skipper et al. ${ }^{\text {a }}$

\begin{tabular}{|c|c|c|c|c|c|}
\hline No. & Site & $x(\AA)$ & $y(\AA)$ & $z(\AA)$ & $q_{i} / e$ \\
\hline 1 & 8 & 2.64 & 0.00 & 3.28 & -0.8 \\
\hline 2 & 8 & 1.32 & 2.28 & 3.28 & -0.8 \\
\hline 3 & 8 & 3.96 & 2.28 & 3.28 & -0.8 \\
\hline 4 & 8 & 0.0 & 0.0 & 1.06 & -1.7175 \\
\hline 5 & 1 & 0.8815 & 0.0 & 1.434 & 0.7175 \\
\hline 6 & 14 & 2.64 & 1.52 & 2.73 & 1.2 \\
\hline 7 & 14 & 0.0 & 3.05 & 2.73 & 1.2 \\
\hline 8 & 8 & 2.64 & 1.52 & 1.06 & -1.0 \\
\hline 9 & 8 & 0.0 & 3.05 & 1.06 & -1.0 \\
\hline 10 & 13 & 4.4 & 1.52 & 0.0 & 3.0 \\
\hline 11 & 13 & 4.4 & -1.52 & 0.0 & 3.0 \\
\hline 12 & 8 & 0.0 & 4.57 & 3.28 & -0.8 \\
\hline 13 & 8 & 3.96 & 6.85 & 3.28 & -0.8 \\
\hline 14 & 8 & 1.32 & 6.85 & 3.28 & -0.8 \\
\hline 15 & 8 & 2.64 & 4.57 & 1.06 & -1.7175 \\
\hline 16 & 1 & 3.5215 & 4.57 & 1.434 & 0.7175 \\
\hline 17 & 14 & 0.0 & 6.09 & 2.73 & 1.2 \\
\hline 18 & 14 & 2.64 & 7.62 & 2.73 & 1.2 \\
\hline 19 & 8 & 0.0 & 6.09 & 1.06 & -1.0 \\
\hline 20 & 8 & 2.64 & 7.62 & 1.06 & -1.0 \\
\hline 21 & 13 & 7.04 & 6.09 & 0.0 & 3.0 \\
\hline 22 & 13 & 7.04 & 3.05 & 0.0 & 3.0 \\
\hline 23 & 8 & 0.88 & 9.14 & -3.28 & -0.8 \\
\hline 24 & 8 & 2.20 & 6.86 & -3.28 & -0.8 \\
\hline 25 & 8 & -0.44 & 6.86 & -3.28 & -0.8 \\
\hline 26 & 8 & 3.52 & 9.14 & -1.06 & -1.7175 \\
\hline 27 & 1 & 2.6385 & 9.14 & -1.434 & 0.7175 \\
\hline 28 & 14 & 0.88 & 7.62 & -2.73 & 1.2 \\
\hline 29 & 14 & 3.52 & 6.09 & -2.73 & 1.2 \\
\hline 30 & 8 & 0.88 & 7.62 & -1.06 & -1.0 \\
\hline 31 & 8 & 3.52 & 6.09 & -1.06 & -1.0 \\
\hline 32 & 8 & 3.52 & 4.57 & -3.28 & -0.8 \\
\hline 33 & 8 & -0.44 & 2.29 & -3.28 & -0.8 \\
\hline 34 & 8 & 2.2 & 2.29 & -3.28 & -0.8 \\
\hline 35 & 8 & 0.88 & 4.57 & -1.06 & -1.7175 \\
\hline 36 & 1 & -0.0015 & 4.57 & -1.434 & 0.7175 \\
\hline 37 & 14 & 3.52 & 3.05 & -2.73 & 1.2 \\
\hline 38 & 14 & 0.88 & 1.52 & -2.73 & 1.2 \\
\hline 39 & 8 & 3.52 & 3.05 & -1.06 & -1.0 \\
\hline 40 & 8 & 0.88 & 1.52 & -1.06 & -1.0 \\
\hline
\end{tabular}

${ }^{\mathrm{a}}$ Reference 4 .

interactions. Ideal and dispersion contributions must be added to evaluate the complete pressure tensor.

\section{B. Simulation cell}

The positions and charges of the sites in the unit cell of the clay, as given by Skipper et al., ${ }^{4}$ are reproduced in Table I of the Appendix. As described in the Appendix, this unit cell is replicated to form the clay sheet we use in the simulations. The lateral dimensions of the clay sheet are $L_{x}$ $=21.12, L_{y}=18.28$, and $L_{z}=6.56 \AA$. Figures $1(\mathrm{a})$ and $1(\mathrm{~b})$ provide a representative view of typical configurations of hydrated clays using one and two clay sheets, respectively. These configurations are repeated in all three spatial directions through periodic boundary conditions. We have adopted the standard minimum image convention for dispersion interactions, as well as adding homogeneous long-range corrections. ${ }^{9}$ To be consistent with the minimum image

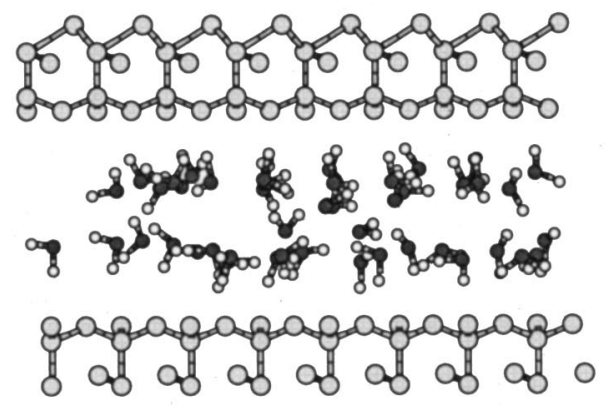

(a)
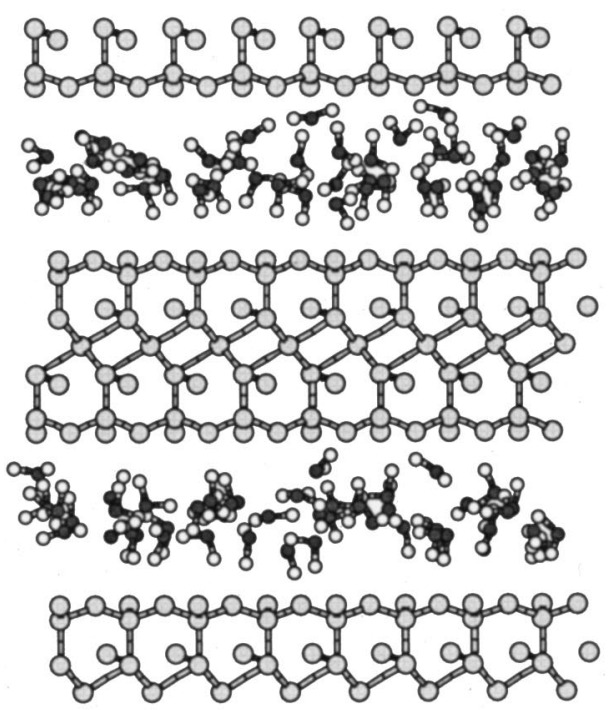

(b)

FIG. 1. (a) Equilibrium configuration of a system with one clay sheet, six sodium ions, and interlayer water. (b) Equilibrium configuration of a system with two clay sheets, twelve sodium ions, and interlayer water. Sodium ions are not shown.

convention, ${ }^{9}$ the short-range interactions are truncated using a spherical cut off, $R_{c}$, which must be no larger than half the smallest dimension of the simulation box.

In previous simulations of clay-water systems, ${ }^{4,5,12}$ a single clay sheet was employed and replicated in the three spatial directions through the use of periodic boundary conditions (in an attempt to mimic the stacked structure commonly present in clay systems). As indicated above, montmorillonite clays may consist of layers stacked upon each other, with the interlayer water and cations between each sheet. Thus, the use of two or more independent clay layers provides a more faithful representation of the physical system than a single layer. Furthermore, using more than one sheet reduces finite size effects and improves statistics. As shown below, the results obtained using one and two clay sheets differ at small basal spacings, whereas the results from two and three clay sheets are virtually identical.

In this work we use two different clay sheets, termed Clay I and Clay II. Given the positions and charges of the mineral pyrophyllite (Appendix), the sodium-based Wyoming montmorillonite Clay I is obtained by isomorphous substitution of trivalent $\mathrm{Al}$ atoms in the positions $(-3.52$, $-3.05,0.00),(7.04,-3.05,0.00),(-3.52,6.09,0.00)$, and 
TABLE II. Interaction parameters corresponding to the MCY model.

\begin{tabular}{cclcc}
\hline \hline Sites & $\mathrm{A}(\mathrm{kcal} / \mathrm{mol})$ & $\mathrm{B}(1 / \AA)$ & $\mathrm{C}(\mathrm{kcal} / \mathrm{mol})$ & $\mathrm{D}(1 / \AA)$ \\
\hline $\mathrm{H}-\mathrm{H}$ & 0.0 & 0.0 & 666.330 & 2.7608 \\
$\mathrm{H}-\mathrm{O}$ & 273.59 & 2.2333 & 14455.4 & 2.9619 \\
$\mathrm{O}-\mathrm{O}$ & 0.0 & 0.0 & 1088213.0 & 5.1527 \\
$\mathrm{H}-\mathrm{Na}$ & 884.23 & 1.9349 & 2051.9 & 2.3609 \\
$\mathrm{O}-\mathrm{Na}$ & 25.948 & 0.77461 & 61888.0 & 4.0849 \\
$\mathrm{H}-\mathrm{Si}$ & 2.137 & 1.22 & 577.23 & 2.1564 \\
$\mathrm{H}-\mathrm{Al}$ & 2.137 & 1.22 & 577.23 & 2.1564 \\
$\mathrm{O}-\mathrm{Si}$ & 1345.8 & 2.2671 & 13061.0 & 3.2037 \\
$\mathrm{O}-\mathrm{Al}$ & 1345.8 & 2.2671 & 13061.0 & 3.2037 \\
$\mathrm{Si}-\mathrm{Na}$ & 1505.4 & 1.8652 & 2164.54 & 2.1209 \\
$\mathrm{Al}-\mathrm{Na}$ & 1505.4 & 1.8652 & 2164.54 & 2.1209 \\
\hline \hline
\end{tabular}

(7.04, 6.09, 0.00) of the octahedral sheet by divalent $\mathrm{Mg}$ atoms. Tetravalent $\mathrm{Si}$ atoms in the tetrahedral sheets with positions $(2.64,1.52,2.73)$ and $(0.88,1.52,-2.73)$ are substituted by trivalent $\mathrm{Al}$ atoms. Clay II is obtained from Clay I by keeping the $\mathrm{Si}$ atom in the position $(0.88,1.52,-2.73)$ and substituting instead the $\mathrm{Si}$ atom in the position $(-9.68$, $1.52,-2.73)$. All positions are given in angstroms. As for the interaction parameters, the set of values proposed by Skipper et al. ${ }^{4}$ for both the MCY and the TIP4P models are reproduced in Tables II and III. Note, however, that in this work we use the set of parameters proposed by Boek et al. ${ }^{12}$ for the TIP4P model, where the exponential contributions are eliminated and the interactions are based on a 12-6 LennardJones potential.

\section{Constant stress simulations}

In constant stress simulations, the stress normal to the surface of the clays, $P_{z z}$, is kept constant while the volume of the system is allowed to fluctuate only in the $z$-direction. At equilibrium, basal spacings oscillate around their average value. In this ensemble, the probability of accepting a trial change in the configuration of the system (through either a volume fluctuation or a molecular displacement), only depends on the change of energy and volume of the system in going from one state or configuration to the other. In general, the energy change in going from an initial state $m$ to a final state $n$ can be written as

$$
\Delta H=\Delta U+P_{z z} \Delta V-\frac{N}{\beta} \ln \left(V_{n} / V_{m}\right),
$$

and the acceptance probability is given by

TABLE III. Interaction parameters corresponding to the TIP4P model. The parameters for $\mathrm{O}-\mathrm{O}$ and $\mathrm{O}-\mathrm{Na}$ interactions are taken from the set proposed by Boek et al. in Ref. 12 .

\begin{tabular}{ccccc}
\hline \hline Sites & $\begin{array}{c}\mathrm{C} \\
(\mathrm{kcal} / \mathrm{mol})\end{array}$ & $\begin{array}{c}\mathrm{D} \\
(1 / \AA)\end{array}$ & $\begin{array}{c}\mathrm{E} \\
\left(\AA^{6} \mathrm{kcal} / \mathrm{mol}\right)\end{array}$ & $\begin{array}{c}\mathrm{F} \\
\left(\AA^{12} \mathrm{kcal} / \mathrm{mol}\right)\end{array}$ \\
\hline $\mathrm{O}-\mathrm{O}$ & 0.0 & 0.0 & 602.71152 & 592840.4 \\
$\mathrm{H}-\mathrm{Na}$ & 2064.0 & 3.394 & 0.0 & 0.0 \\
$\mathrm{O}-\mathrm{Na}$ & 0.0 & 0.0 & 422.7847 & 90557.854 \\
$\mathrm{Si}-\mathrm{Na}$ & 951.71 & 2.1286 & 0.0 & 0.0 \\
$\mathrm{Al}-\mathrm{Na}$ & 951.71 & 2.1286 & 0.0 & 0.0 \\
\hline \hline
\end{tabular}

$$
P_{\mathrm{acc}}=\min \{1, \exp (-\beta \Delta H)\},
$$

where $\Delta U$ is the change in potential energy, $\Delta V$ is the change in volume, and $V_{m}$ and $V_{n}$ are the volumes of the simulation box before and after the trial move, respectively. The inverse temperature is $\beta^{-1}=k_{B} T$, where $k_{B}$ is Boltzmann's constant and $T$ is the absolute temperature. The trial Monte Carlo moves employed in this work are displacement and/or rotation of water molecules and sodium ions, or a volume change in which only the $z$-components of the center-of-mass of each molecule are scaled. Note that the number of water molecules in the system is kept constant.

\section{Constant chemical potential simulations}

In situations where expanding clay minerals are in contact with water, molecules are exchanged between the supernatant solution and the interlayer space until chemical equilibrium is established. To simulate this exchange of water, we also conduct simulations in the grand canonical ensemble. In these calculations, water molecules are allowed to move in and out of the simulation box, while the number of sodium ions is kept constant. The Monte Carlo trial moves now consist of displacements and/or rotation of water molecules and sodium ions, and deletion or insertion of water molecules. The latter moves, however, can be difficult because water in the interlayer can reach relatively high densities. In order to improve the insertion/deletion rate, a rotational-bias insertion method is employed. ${ }^{16,17}$ In this method, a water molecule is first inserted at a random position in the volume $V=L_{x} L_{y} L_{z}$. Once inserted, $k$ random orientations are generated, and the change in energy $\delta U_{i}, i$ $=1, \ldots, k$, resulting from the insertion of the molecule in the $i$ th orientation is calculated and stored. The $j$ th orientation is then selected with probability $P_{j}$ given by

$$
P_{j}=\frac{\exp \left[-\delta U_{j} / k_{B} T\right]}{\sum_{i=1}^{k} \exp \left[-\delta U_{i} / k_{B} T\right]}=\frac{w_{j}}{\sum_{i=1}^{k} w_{i}} .
$$

The trial insertion of the molecule in the $j$ th orientation is then accepted with probability

$$
P_{\mathrm{acc}}=\min \left[1, \frac{1}{k P_{j}} \exp \left(-\beta\left[\delta U_{n m}+\ln \left(\frac{z V}{N+1}\right)\right]\right)\right],
$$

where $\delta U_{n m}$ is the change in the energy of the system due to the insertion of the molecule in that particular orientation, and $z$ is the activity, which is related to the chemical potential $\mu$ through $z=\exp (\beta \mu) / \Lambda^{3}$, where $\Lambda$ is the thermal length of the molecule. ${ }^{9}$ As for the destruction of a water molecule, $(k-1)$ random orientations are generated and the factor

$$
P_{j=1}=\frac{w_{j=1}}{\sum_{i=1}^{k} w_{i}}
$$

is calculated. The trial destruction of a molecule is accepted with probability

$$
P_{\text {acc }}=\min \left[1, k P_{j=1} \exp \left(-\beta\left[\delta U_{n m}+\ln \left(\frac{N}{z V}\right)\right]\right)\right] .
$$




\section{E. Simulation details}

All of our $N P_{z z} T$ simulations were performed at $T$ $=300 \mathrm{~K}$ and $P_{z z}=1 \times 10^{5} \mathrm{~Pa}=1$ bar. At the beginning of the simulations, an ordered configuration was prepared, with a basal spacing chosen such that the volume of the simulation box decreased in the initial steps of the simulation. To generate an initial configuration of a system with one clay sheet, the cations are placed randomly in the middle of the interlayer. The water molecules are then placed in a squared array on both sides of the plane defined by the cations. These arrays are located at half the distance between the plane containing the cations and the surface of the clay. This configuration is replicated for systems with two or three clay sheets. At least $2 \times 10^{6}$ Monte Carlo steps were used for the equilibration period. After equilibration, the simulations proceeded for at least $2 \times 10^{6}$ Monte Carlo steps. Data for the averages were collected every $N_{c} \times N_{w}$ steps, where $N_{c}$ is the number of clays and $N_{w}$ is the number of water molecules per clay in the simulation.

For simulations in the $\mu V T$ ensemble, the chemical potential $\mu$ of bulk water was calculated by performing NPT (i.e., constant pressure and temperature) simulations of bulk water at $T=300 \mathrm{~K}$ and at $P=1 \mathrm{bar}$. In this ensemble, the Widom insertion method was used on a system of 216 TIP4P water molecules, to yield $\beta \mu=-17.408$. During the simulations, the Monte Carlo trial moves (displacement, insertion, and deletion of molecules) were taken with a 2:1:1 proportionality. To bias insertion and deletion moves, $k=20$ trial orientations were used.

As indicated previously, we used Ewald summations to evaluate the electrostatic interactions. In all the simulations, the parameter $\alpha$ was taken to be $\alpha=5.6 / 2 R_{c}$, and the reciprocal contributions to the electrostatic energy were truncated at the maximum reciprocal vector, $k_{\max }$, defined as $k_{\max }=5$ $\times k_{0}$, where $k_{0}=2 \pi / L_{\min }$ and $L_{\min }$ is the minimum length of the simulation box. Furthermore, short-range interactions were truncated at a distance smaller than, or equal to $L_{\min } / 2$. Dispersion interactions were corrected using the standard long-range corrections for homogeneous fluids. ${ }^{9}$ This procedure differs from that taken by other authors where no Ewald sums were employed, or where a cutoff larger than that allowed by the dimensions of the simulation box was implemented. ${ }^{4-6,12}$

\section{RESULTS AND DISCUSSION}

\section{A. $N P_{z z} T$ simulations}

In previous simulations of clay systems, only one clay sheet has been used to study the stable basal spacing and the swelling behavior of clays. In order to investigate whether a single clay sheet is sufficient, we performed simulations at constant stress using one, two, and three clay sheets. The water model for these calculations was TIP4P, and the clay was Clay I. The predicted basal spacings for 40 water molecules per clay are $D_{1}=14.80 \AA, D_{2}=12.57 \AA$, and $D_{3}$ $=12.56 \AA$ for one, two, and three clay sheets, respectively. For 64 water molecules, we get $D_{1}=14.87 \AA, D_{2}=D_{3}$ $=14.86 \AA$. These results show that the basal spacing of sodium smectites does indeed depend on the number of clay

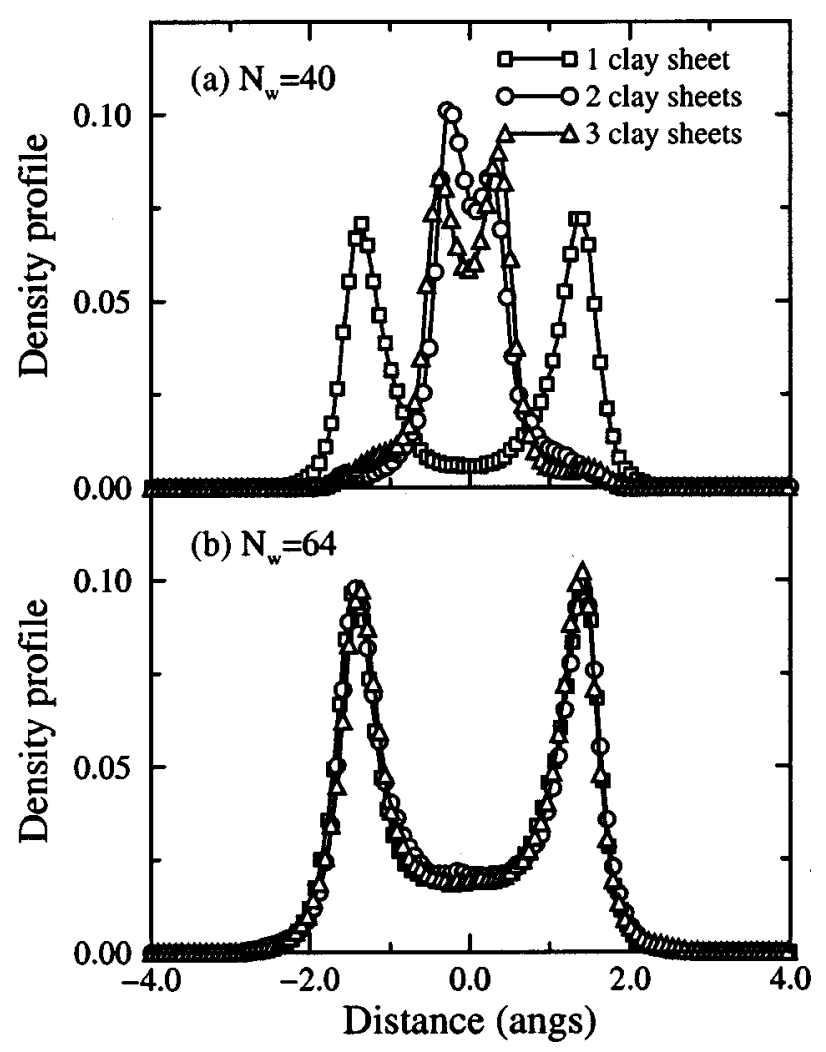

FIG. 2. (a) Density profiles of water oxygens for $N_{w}=40$, using one, two, and three clay sheets. (b) Density profiles of water oxygens for $N_{w}=64$, using one and two clay sheets. Clay I is used. Simulations were conducted at $T=300 \mathrm{~K}$ and $P_{z z}=1$ bar.

layers used in the simulations, particularly at small distances. The distribution of water molecules and sodium ions in the interlayer also depends on the number of clay sheets. This is illustrated by Figs. 2(a) and 2(b), where we show density profiles (in units of $\AA^{-3}$ ) of water oxygens for $N_{w}=40$ and $N_{w}=64$.

For 40 water molecules per clay and for one clay sheet, we see that the density profile of water oxygens consists of two peaks, indicating formation of two layers of water in the interlayer space. On the other hand, when two or three clay sheets are used, the density profile indicates that most of the water molecules are confined to a single layer. For 64 water molecules per clay, however, the density profiles simulated with one and two clay sheets are in closer agreement. In this case, the results indicate the formation of two layers of water. Based on these results, we choose to simulate montmorillonite systems with at least two clay sheets.

Figure (3) shows the density profiles of water oxygens, along with the density profiles of water hydrogens and sodium ions for a system with two Clay I sheets. For 40 water molecules per clay, we see that the distribution of water hydrogens has a three-peak symmetric structure, with a maximum at the center of the clay. The sodium ions are distributed around the oxygens, but preferentially concentrated in the regions where the concentration of water hydrogens is lower. Furthermore, we find that the sodium ions are not fully solvated since they are not completely surrounded by oxygen atoms. These findings should be contrasted with 


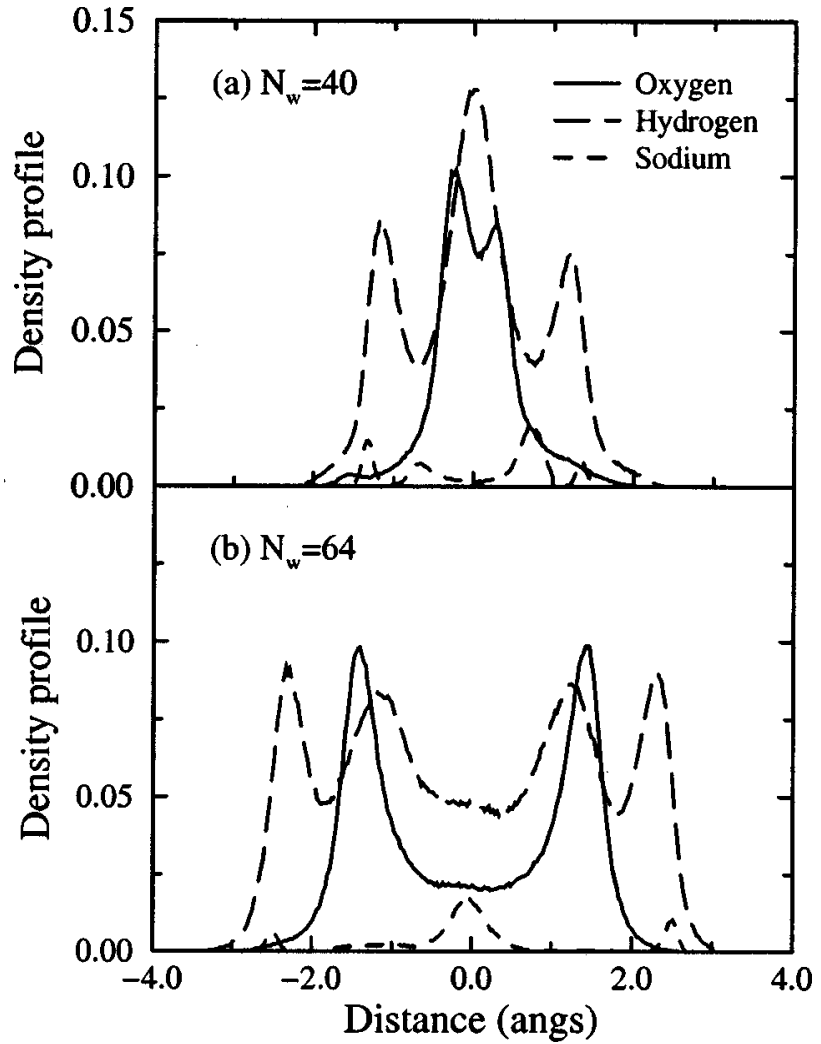

FIG. 3. Distribution of the oxygens, hydrogens, and sodiums from an $N P_{z z} T$ simulation at $P_{z z}=1$ bar, $T=300 \mathrm{~K}$, and using two Clay I sheets. (a) $N_{w}=40$. (b) $N_{w}=64$

those for $N_{w}=64$ and $N_{w}=200$ [Fig. (4)], where most of the sodium ions are clearly surrounded by water oxygens. Some of them, however, are as close as $1.7 \AA$ from the surface of the clay sheets (approximately), as indicated by the small peaks of their density profiles [Figs. 3(a) and 3(b)]. The density profiles represent an average over the positions of all atoms; our results suggest that, on the average (and for the molecular model and the conditions employed here), it is more likely for a hydrogen atom than for a cation to be closer to the surface of the clay. The large degree of overlap between the oxygen and sodium density profiles, indicates

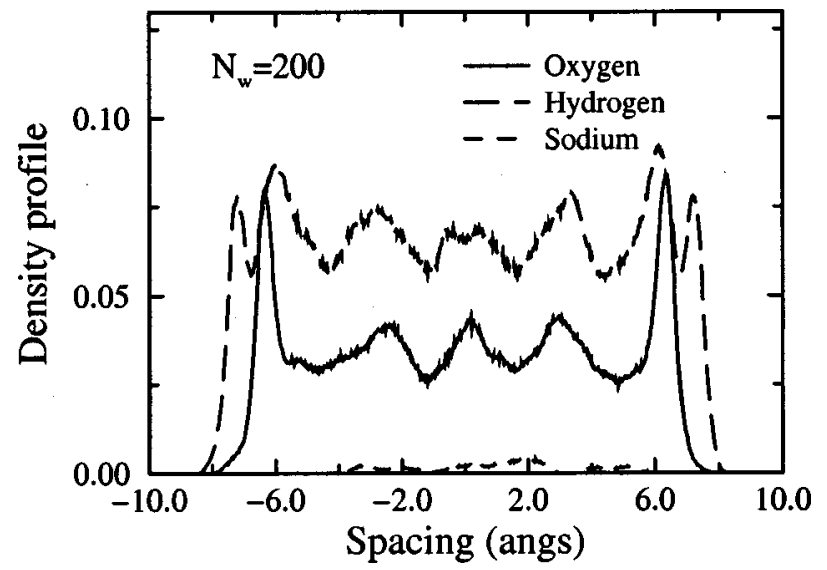

FIG. 4. Distribution of the oxygens, hydrogens, and sodiums in an $N P_{z z} T$ simulation with $N_{w}=200, P_{z z}=1 \mathrm{bar}$, and $T=300 \mathrm{~K}$. The average spacing is $24.72 \AA$.

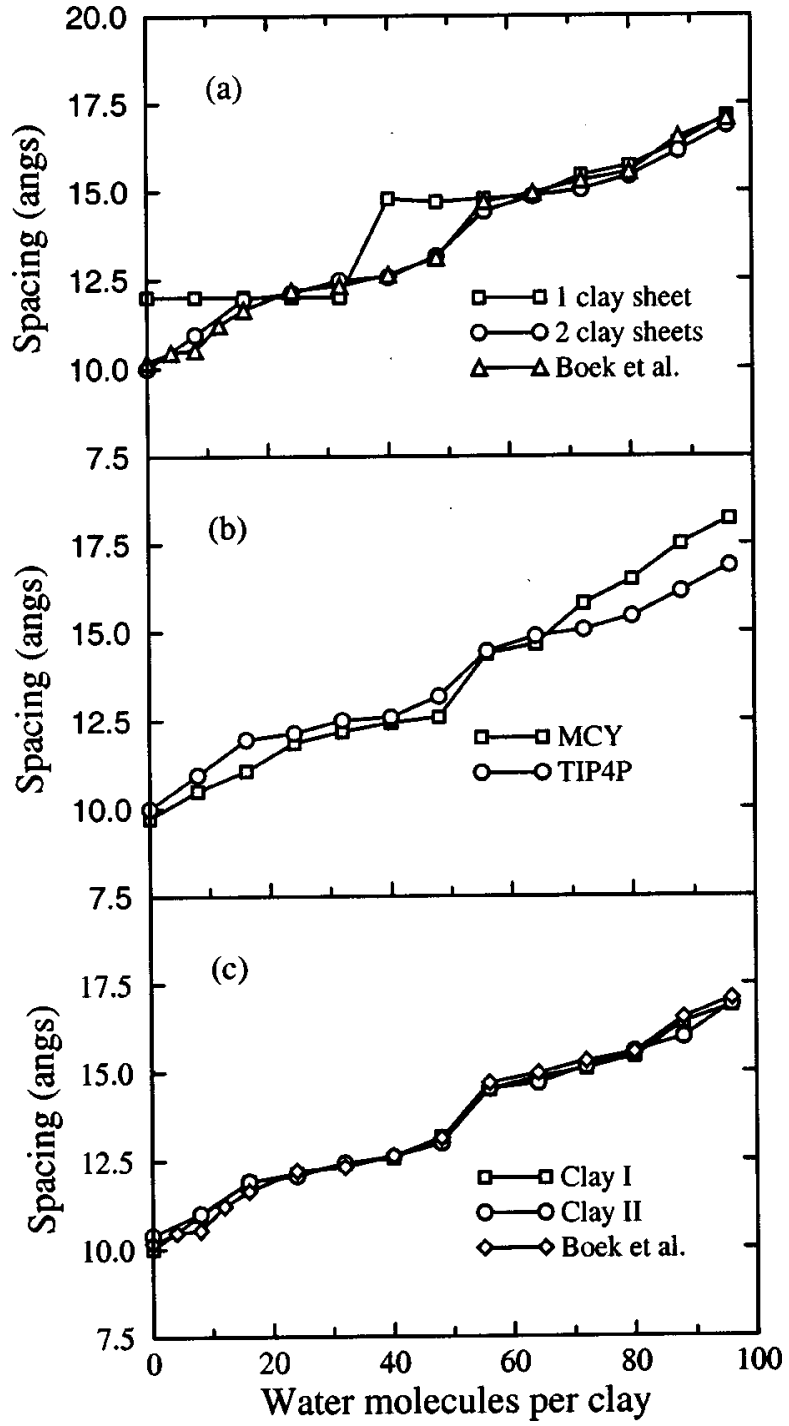

FIG. 5. (a) Swelling curve for one and two clay sheets. They are compared to the results reported by Boek et al. (b) Swelling curve for the TIP4P and the MCY water models. In (a) and (b) Clay I is employed. (c) Swelling for Clays I and II using the TIP4P water model and two clay sheets. The simulations are conducted at $P_{z z}=1 \mathrm{bar}$ and $T=300 \mathrm{~K}$.

that most of the cations are hydrated, which should facilitate ionic exchange with surrounding fluids. These results are consistent with those of Skipper et al. ${ }^{5}$ for clay-water systems having compositions similar to ours. Our results also support the experimental data presented by Sposito et al. $;^{18}$ these authors report results of infrared spectroscopic experiments on Na-saturated Wyoming montmorillonite which indicate that, as the water content in the interlayer increases, the exchangeable cations move out of the cavities in the clay surface to position themselves in the interlayer space, where they are solvated by water molecules.

We now present our simulation results for the swelling of our model clay, i.e., the behavior of the basal spacing as a function of the number of water molecules in the interlayer. (See Table IV.) We do this for one and two clay sheets using Clay I and the TIP4P water model; the results are shown in Fig. 5(a), where the results reported by Boek et al. in Ref. 12 are also included. We see here that, at small basal spacings, 
the results predicted when using one clay sheet differ from those predicted with two clay sheets. The results of Boek et al. differ from ours at small values of $N_{w}$, but are otherwise consistent with our findings.

Figure 5(b) shows a comparison of the swelling for the TIP4P and the MCY water models. From the figure, we see that for small amounts of water in the system, the MCY model predicts smaller basal spacings than the TIP4P. At higher values of water content, the predictions of the basal spacings for the MCY model are larger than those predicted by the TIP4P model. In order to estimate the effects on the swelling due to the specific substitutions of atoms in the clay sheet, we also calculated the swelling of Clay II and compared it with the corresponding prediction for Clay I. The distribution of the substitutions in Clay II is similar to those in Clay I; they differ only in the location of one of the substitutions in the tetrahedral sheet. This comparison is presented in Fig. 5(c). As illustrated in the figure, the results for the swelling of Clay II are very similar to those for Clay I. This indicates that, at least for substitutions in the tetrahedral sheets, the effect of such substitutions has a minor effect in the swelling of the system.

\section{B. Grand canonical ( $\mu V T)$ simulations}

When clay minerals are in contact with pure water, the equilibrium properties of the clay-water system, such as the interlayer water content, the density profiles, and the stable basal spacings are determined by $\mu$, the chemical potential of water. We therefore begin by estimating the chemical potential of the molecules whose content in the simulation cell is allowed to fluctuate. For consistency, the chemical potential used in our simulations was not the experimental value of $\mu$, but the value obtained from NVT (i.e., constant volume and temperature) and NPT simulations of the water models employed here at $T=300 \mathrm{~K}$ and $P=1$ bar. We performed NPT simulations of bulk water using the TIP4P model, with $N$ $=216$ water molecules in the simulation cell at $T=300 \mathrm{~K}$ and $P=1$ bar. The chemical potential was determined by inserting approximately $1 \times 10^{9}$ water molecules. We obtained $\beta \mu=-17.408$. This is the value used in our grand canonical calculations.

We used the chemical potential of bulk TIP4P water and conducted grand canonical simulations using two type-I clay sheets. In each of these simulations, the basal spacing was kept fixed and the system was allowed to equilibrate under the specified chemical potential of bulk water. Once at equilibrium, the total pressure normal to the clays, or the disjoining pressure, $P_{z z}$, was calculated, as opposed to the disjoining pressure of water molecules alone.

Our results for $P_{z z}$ are shown in Fig. 6(a). The behavior of $P_{z z}$ is oscillating in nature. The mechanically stable basal spacings are determined from the intersections of this curve (with negative slope) with a line corresponding to the external pressure ( 1 bar in this work). ${ }^{8}$ The stable distances for TIP4P molecules are therefore $D_{1}=10.2 \AA, D_{2}=12.2 \AA$, $D_{3}=15.7 \AA$. A fourth stable spacing could also exist at 18.7 $\AA$ although the statistical noise of our results is too large to make more definite conclusions about that particular value.

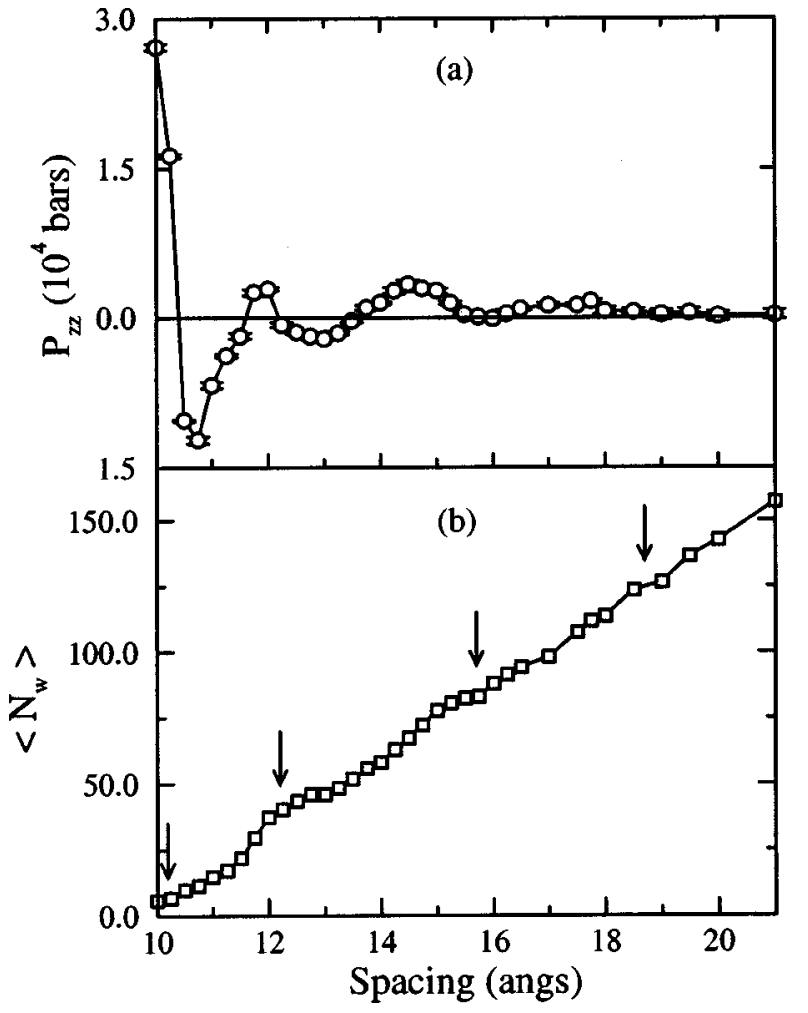

FIG. 6. Simulation results using the grand canonical ensemble with temperature $T=300 \mathrm{~K}$ and chemical potential $\beta \mu=-17.408$. (a) Normal component of the pressure tensor. (b) Average number of water molecules per clay.

These spacings are in reasonable agreement with experiments, ${ }^{19-22}$ which indicate that $D_{1}^{\exp }=9.6-10.1 \AA$, $D_{2}^{\text {exp }}=12.0-12.6 \AA, \quad D_{3}^{\exp }=15.0-15.55 \AA, \quad$ and $\quad D_{4}^{\exp }$ $=18.0-18.1 \AA$. In similar work, ${ }^{6}$ the stable distances reported for an ideal montmorillonite and for the MCY model were $D_{1}=9.7 \AA, \quad D_{2}=12.0 \AA, \quad D_{3}=15.5 \AA, \quad$ and $D_{4}$ $=18.3 \AA$. Our results for the swelling of the clay system are presented in Fig. 6(b). Here, the average number of water molecules per clay is plotted as a function of the basal spacing. Note that the swelling is not uniform, and that there are regions, albeit small, where increasing $D$ has little effect on the water content. These regions are located around the stable clay separations. Similar trends were found in Ref. 6 , although in that work the planar regions were wider. However, our findings contradict those of Ref. 6 where, based on calculations of the stable basal spacings, it was proposed that the swelling proceeds from the dry state through the formation of odd numbers (one, three, and then five) of welldefined layers of water. Our results do not show any evidence of formation of only odd numbers of layers. Reference 6 does not make any mention of using special methods to handle long-range interactions; it is conceivable that some of the discrepancies between that work and ours could be due to the truncation of long-range Coulomb interactions in Ref. 6.

In Fig. 7(a) we present the density profiles for the water oxygens at our predicted stable basal spacings. At $D=10.2 \AA$ the small amount of water is confined to the clay cavities. From the figure we notice that we obtain a one-layer and a two-layer structure of water molecules for $D=12.2$ 


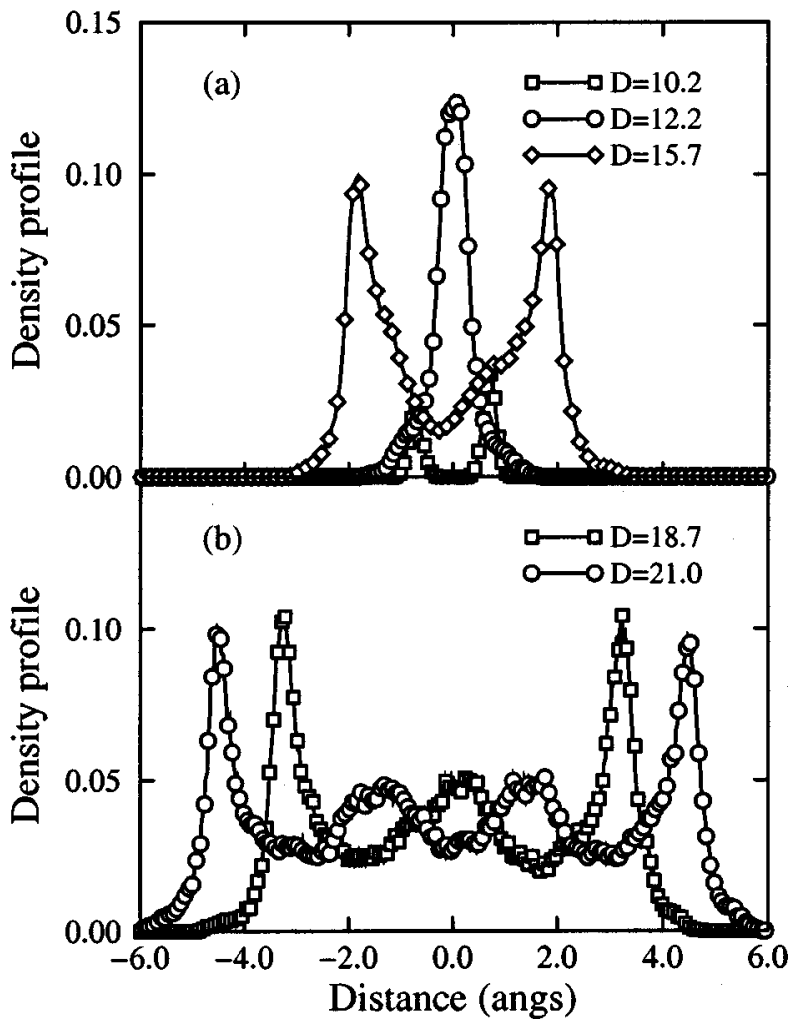

FIG. 7. Distribution of water oxygens for the different stable basal spacings determined in the previous figure. (a) Density profiles for the stable basal spacings shown in Fig. 6(a). (b) Density profiles for large values of the basal spacing.

and $D=15.7 \AA$, respectively. For larger basal spacings, we see the formation of three and four layers of water for $D$ $=18.7$ and $D=21.0$, respectively, as illustrated in Fig. 7(b).

\section{SUMMARY}

We have presented results of Monte Carlo simulations of hydrated clays using an atomistic representation of the Wyo-

TABLE IV. Calculated basal spacings as function of water content. In column 1 are the number of water molecules per clay used in the simulations. In column 2 are the calculated basal spacings using Clay I and the TIP4P water model. In column 3 are the calculated basal spacings using Clay II and the TIP4P water model. In column 4 are the calculated basal spacings using Clay I and the MCY water model. The last column presents the basal spacings calculated by Boek et al.

\begin{tabular}{rrcrc}
\hline \hline$N_{w}$ & $D_{\mathrm{I}}^{\mathrm{TIP4P}}(\AA)$ & $D_{\text {II }}^{\mathrm{TIP} 4 \mathrm{P}}(\AA)$ & $D_{\mathrm{I}}^{\mathrm{MCY}}(\AA)$ & $D_{\text {Boek }}$ \\
\hline 0 & 9.993 & 10.394 & 9.729 & 10.17 \\
8 & 10.944 & 11.119 & 10.501 & 10.52 \\
16 & 11.947 & 11.867 & 11.067 & 11.64 \\
24 & 12.129 & 12.073 & 11.864 & 12.20 \\
32 & 12.499 & 12.472 & 12.166 & 12.32 \\
40 & 12.571 & 12.562 & 12.421 & 12.64 \\
48 & 13.173 & 13.092 & 12.589 & 13.13 \\
56 & 14.450 & 14.557 & 14.380 & 14.70 \\
64 & 14.869 & 14.888 & 14.649 & 14.96 \\
72 & 15.061 & 15.135 & 15.797 & 15.30 \\
80 & 15.433 & 15.560 & 16.473 & 15.55 \\
88 & 16.138 & 16.170 & 17.497 & 16.54 \\
96 & 16.869 & 17.108 & 18.192 & 17.07 \\
\hline \hline
\end{tabular}

ming sodium montmorillonite. Under simulations at constant normal stress, we examined the swelling behavior and the evolution of the interlayer density profiles of water and sodium ions. In particular, we found that the interlayer water evolves from a single layer to two and then three water layers, as the water content in the interlayer increases. Further increments in the water content eventually yield formation of additional water layers (four and five for 160 and 200 water molecules per clay, respectively). It was also shown in this work that, at the conditions studied here, the sodium ions have a strong tendency to be hydrated and to drift away from the surface of the clay. This is consistent with the simulations of Skipper et al..$^{5}$ and with the experimental work of Sposito et al. ${ }^{18} \mathrm{We}$ found this trend in all the basal spacings considered in this work. This contrasts previous work, ${ }^{6}$ where the sodium ions were in general reported to be directly next to the clay surface.

Simulations in the grand canonical ensemble allowed us to determine the stable basal spacings and the swelling behavior of a sodium montmorillonite clay-water model. We found that, within the dispersion in the experimental data and the statistical uncertainty of our simulations, the stable basal spacings determined from the pressure tensor are in good agreement with experiments. We also found that the swelling of the clay-water system is not uniform, with regions where increments in the basal spacing lead to minor increments in the water content. These regions correspond to the vicinity of the stable basal spacings.

\section{ACKNOWLEDGMENTS}

This work was partially supported through an NSFCONACyT collaborative grant. The authors are also grateful to the Mexican Petroleum Institute for partial support.

\section{APPENDIX}

The positions and charges of the sites in the unit cell of clay mineral pyrophyllite are given by Skipper et al., ${ }^{4}$ which are reproduced in Table I. As indicated in the introduction, we used the (clay termed) Wyoming montmorillonite whose unit cell formula is $\mathrm{Na}_{0.750} n \mathrm{H}_{2} \mathrm{O}\left(\mathrm{Si}_{7.75} \mathrm{Al}_{0.25}\right)\left(\mathrm{Al}_{3.5} \mathrm{Mg}_{0.5}\right) \mathrm{O}_{20}(\mathrm{OH})_{4}$.

To obtain Wyoming montmorillonite from pyrophyllite, one out of eight aluminum atoms in the octahedral sheet are substituted by magnesium atoms, and one out of 32 silica atoms are replaced by aluminum atoms. To obtain the actual piece of clay we used in this work and named Clay I, we take the sequence of the atoms as indicated in Table I, and duplicated it in the $x$-direction by displacing the atoms by a distance $D_{x}=5.28 \AA$; the resulting structure is then duplicated by displacing it a distance $2\left(D_{x}\right)$ in the $x$-direction. After this, we duplicate again the resulting structure but now in the $y$-direction by displacing the structure by $2 \times(4.57) \AA$ in the $y$-direction. The resulting structure is displaced by $\left(L_{x} / 2, L_{y} / 2\right)$ and then periodic boundary conditions are applied to center the structure in our simulation cell. At the end, a patch of clay of lateral dimensions $L_{x}=21.12 \AA, L_{y}$ $=18.28 \AA, L_{z}=6.56 \AA$ is obtained. We then substitute the aluminum atoms in the positions $(-3.52,-3.05,0.00)$, 
(7.04, -3.05, 0.00), (-3.52, 6.09, 0.00), and (7.04, 6.09, $0.00)$ in the octahedral sheet by $\mathrm{Mg}$ atoms, whereas the $\mathrm{Si}$ atoms in the tetrahedral sheets with positions $(2.64,1.52$, $2.73)$, and $(0.88,1.52,-2.73)$ were substituted with $\mathrm{Al}$ atoms. To obtain Clay II, we take Clay I with the substitution in $(0.88,1.52,-2.73)$ now replaced by a substitution in $(-9.68,1.52,-2.73)$.

${ }^{1}$ B. Velde, Origin and Mineralogy of Clays (Springer, New York, 1995).

${ }^{2}$ H. van Olphen, Clay Colloid Chemistry (Wiley, New York, 1977).

${ }^{3}$ A. C. D. Newman, Chemistry of Clays and Clay Minerals (Mineralogical Society, London, 1987).

${ }^{4}$ N. T. Skipper, F. Chou Chang, and G. Sposito, Clays Clay Miner. 43, 285 (1995).

${ }^{5}$ N. T. Skipper, G. Sposito, and F. Chou Chang, Clays Clay Miner. 43, 294 (1995).

${ }^{6}$ S. Karaborni, B. Smit, W. Heidug, J. Urai, and E. van Oort, Science 271, 1102 (1996).

7 (a) A. Delville, J. Phys. Chem. 97, 9703 (1993); (b) 99, 2033 (1995).

${ }^{8}$ R. M. Shroll and D. E. Smith, J. Chem. Phys. 111, 9025 (1999).

${ }^{9}$ (a) M. P. Allen and D. J. Tildesley, Computer Simulation of Liquids (Clar- endon, Oxford, 1986); (b) D. Frenkel and B. Smit, Understanding Molecular Simulations (Academic, New York, 1996).

${ }^{10}$ W. L. Jorgensen, J. Chandrasekhar, and J. D. Madura, J. Chem. Phys. 79, $926(1983)$

${ }^{11}$ G. C. Lie, E. Clementi, and M. Yoshimine, J. Chem. Phys. 64, 2314 (1976).

${ }^{12}$ E. S. Boek, P. V. Conevey, and N. T. Skipper, Langmuir 11, 4629 (1995).

${ }^{13}$ S. W. de Leeuw, J. W. Perram, and E. R. Smith, Proc. R. Soc. London, Ser. A 373, 27 (1980).

${ }^{14}$ J. Alejandre, D. J. Tildesley, and G. A. Chapela, J. Chem. Phys. 102, 4574 (1994).

${ }^{15}$ S. Nosé and M. L. Klein, Mol. Phys. 50, 1055 (1983).

${ }^{16}$ R. F. Cracknell, D. Nicholson, and N. G. Parsonage, Mol. Phys. 71, 931 (1990).

${ }^{17}$ J. J. dePablo, M. Laso, and U. W. Suter, J. Chem. Phys. 96, 6157 (1992).

${ }^{18}$ G. Sposito, R. Prost, and J.-P. Gaultier, Clays Clay Miner. 31, 9 (1963).

${ }^{19}$ D. J. Cebula, R. K. Thomas, S. Middleton, R. H. Ottewil, and J. W. White, Clays Clay Miner. 27, 39 (1979).

${ }^{20}$ M. H. Fu, Z. Z. Zhang, and P. F. Low, Clays Clay Miner. 38, 5 (1990).

${ }^{21}$ J. M. Trillo, J. Poyato, M. M. Tobías, and M. A. Castro, Clays Clay Miner. 25, 485 (1990)

${ }^{22}$ J. M. Cases, I. Bérend, G. Besson, M. François, J. P. Uriot, F. Thomas, and J. E. Poirier, Langmuir 8, 2730 (1992). 